\title{
Biofilm development by potentially pathogenic non-pigmented rapidly growing mycobacteria
}

\author{
Jaime Esteban*1, Nieves Z Martín-de-Hijas1, Teemu J Kinnari1, \\ Guillermo Ayala ${ }^{2}$, Ricardo Fernández-Roblas ${ }^{1}$ and Ignacio Gadea ${ }^{1}$
}

\author{
Address: ${ }^{1}$ Department of Clinical Microbiology, Fundación Jiménez Díaz-UTE, Madrid, Spain and ${ }^{2}$ Department of Statistics, University of \\ Valencia, Valencia, Spain \\ Email: Jaime Esteban* - jestebanmoreno@gmail.com; Nieves Z Martín-de-Hijas - bastet.sekhmet@gmail.com; \\ Teemu J Kinnari - teemu.j.kinnari@helsinki.fi; Guillermo Ayala - guillermo.ayala@uv.es; Ricardo Fernández-Roblas - rfernandez@ fjd.es; \\ Ignacio Gadea - igadea@fjd.es \\ * Corresponding author
}

Published: 17 October 2008

BMC Microbiology 2008, 8:184 doi:10.1|86/|47|-2/80-8-184

This article is available from: http://www.biomedcentral.com//47/-2/80/8//84

(c) 2008 Esteban et al; licensee BioMed Central Ltd.

This is an Open Access article distributed under the terms of the Creative Commons Attribution License (http://creativecommons.org/licenses/by/2.0), which permits unrestricted use, distribution, and reproduction in any medium, provided the original work is properly cited.
Received: 8 April 2008

Accepted: 17 October 2008

\begin{abstract}
Background: A study to evaluate the biofilm-development ability in three different media (Middlebrook 7H9, sterile tap water and PBS-5\% glucose) was performed with 19 collection strains from 15 different species on non-pigmented rapidly growing mycobacteria (NPRGM). A microtiter plate assay was developed to evaluate the percentage of covered surface of the microtiter plate wells in different days from day I to day 69.

Results: All strains were able to develop biofilm in all the tested media. Middlebrook $7 \mathrm{H} 9$ showed the fastest growth, followed by sterile tap water and PBS-5\% glucose. A sigmoid growth curve was detected in all the strains both in Middlebrook $7 \mathrm{H} 9$ and in sterile tap water. A difference could be detected for Mycobacterium abscessus in tap water, where it showed faster growth than all the other strains.

Conclusion: Biofilm development seems to be a property of all the species of NPRGM and it depends on the nutrients present in the medium. The microtiter plate assay described here is a useful tool to evaluate differences in biofilm development among the different species of rapidly growing mycobacteria.
\end{abstract}

\section{Background}

Non-pigmented rapidly growing mycobacteria (NPRGM) are among the most commonly isolated species of nontuberculous mycobacteria in clinical laboratories. Although most of the members of this group have been described as the cause of human infections, Mycobacterium abscessus, Mycobacterium fortuitum and Mycobacterium chelonae are the most frequently isolated species in these syndromes $[1,2]$. Among the broad spectrum of these infections, nosocomial diseases are the most important because they may have devastating outcomes [1,3]. Many of these infections are related to implantable medical devices.

NPRGM are also environmental organisms that can be found in many habitats $[4,5]$. Several studies have shown that these organisms can be recovered from different water sources, including biofilms present in plumbing systems. Biofilms are considered important in devicerelated infections due in part to their increased resistance to antimicrobials $[6,7]$. However, despite their impor- 
tance as human pathogens, there are only a few in vitro studies about NPRGM. In this study we report the results of a series of experiments aimed to evaluate the ability of different strains of NPRGM (including all the clinically relevant species) to develop biofilm under different nutrient conditions, and the relationship of biofilm development with the presence of sliding motility among these strains.

\section{Methods \\ Strains}

The following strains were used in the experiments: $\mathrm{Myco}$ bacterium fortuitum ATCC $6841 \mathrm{~T}$ and ATCC 13756, Mycobacterium chelonae ATCC 19235 and ATCC 35752T, Mycobacterium abscessus DSM 44196T, Mycobacterium peregrinum ATCC 14467T, Mycobacterium mucogenicum DSM 44124, Mycobacterium septicum ATCC 700731T, Mycobacterium immunogenum ATCC 700505T, Mycobacterium mageritense ATCC 700351T, Mycobacterium porcinum ATCC 33776T, Mycobacterium senegalense NCTC 10956T, Mycobacterium elephantis DSM 44368T, Mycobacterium smegmatis ATCC 607, ATCC 19420T and ATCC 14468, Mycobacterium goodii ATCC 700504T, Mycobacterium alvei ATCC 51304T, and Mycobacterium brumae ATCC 51384T. All strains were maintained frozen at $-20^{\circ} \mathrm{C}$ until the experiments were performed.

\section{Sliding motility test}

One colony of each mycobacteria was put in the centre of a plate of motility medium, consisting in Middlebrook $7 \mathrm{H} 9$ with $0.3 \%$ agar without supplements. The inoculated media were incubated at $37^{\circ} \mathrm{C}$ in a $5 \% \mathrm{CO}_{2}$ atmosphere during 2 weeks [8]. The diameter of the bacterial growth was measured at days 4, 8, 12 and 16 using a digital caliper.

\section{Biofilm development test}

After thawing, mycobacteria were checked for purity, inoculated on Middlebrook $7 \mathrm{H} 9$ broth and incubated at $30^{\circ} \mathrm{C}$ during 5 days. These broth cultures were centrifuged at $3000 \times \mathrm{g}$, washed with sterile phosphate buffered saline (PBS) and resuspended and calibrated to a $0.5 \mathrm{McF}$ arland Standard with PBS. Ninety-six well sterile flat-bottom tissue-culture treated polystyrene microtiter plates (Costar, USA) were inoculated with $100 \mu$ l of the suspension. Plates were incubated at $37^{\circ} \mathrm{C}$ during 30 minutes. The inoculum was then removed with a sterile Pasteur pipette. The wells were washed with sterile PBS and $100 \mu$ l of the following media were added for all the strains: Middlebrook 7 H9, PBS- $5 \%$ glucose, PBS- $5 \%$ glucose- $0.5 \%$ glycerol and filter sterilized tap water. Plates were placed on an orbital shaker $(80 \mathrm{rpm})$ and incubated at ambient room temperature for 69 days. The media were replaced on days $1,4,7,11,14,18,21,25,28,32,35,39,41,44$, $47,51,54,58,61,65$ and 69 . On days $1,4,7,11,21,28$,
$35,41,47,54,61$ and 69 , one well was washed with sterile distilled water and stained with basic fuchsine during 30 minutes, washed and decoloured for 10 seconds with absolute ethanol. The stained well was then photographed (3-4 images/well) at low magnification $(10 \times)$ using a Leitz DM IL inverted microscope (Leica, Germany) with an attached Nikon Coolpix 8400 digital camera (Nikon, Japan). Each strain was tested at least two times in different experiments.

To check the viability of mycobacteria, randomly selected wells were analyzed by inoculating the culture medium onto tryptic soy agar-5\% blood agar plates, which were incubated at room temperature during 3 days. All the experiment was repeated when a contamination was detected.

\section{Data analysis}

Digital photographs obtained from the stained wells were analysed with the Image J software (National Institute of Health, Bethesda, MD, USA) to evaluate the surface covered by the biofilm. The proportion of surface covered by biofilm at each time point was used to construct a growth curve of the biofilm. The experiment was stopped when growth covered $100 \%$ of the surface.

\section{Confocal laser scanning microscopy (CLSM)}

To evaluate the biofilm development, we randomly selected 4 strains (M. fortuitum ATCC 6841T, M. septicum ATCC 700731T, M. smegmatis ATCC 19420 and M. immunogenum ATCC 700505T) to be analysed by using confocal laser scanning microscopy (CLSM) using the following protocol:

One $\mathrm{ml}$ of a $0.5 \mathrm{McF}$ arland turbidity inoculum was inoculated onto $6 \times 4$ well-plates (Nunc, USA) with a polystyrene plastic disc (Nunc, USA) at the bottom of each well. Plates were then incubated at room temperature using the same protocol that microtiter plates with Middlebrook 7H9 as culture medium. On days 7, 14 and 21, the medium was removed, and the plastic discs were stained with the Live/dead ${ }^{\odot}$ BacLight $^{\oplus}$ viability stain (Invitrogen, Eugene, OR, USA) according to instructions provided by the manufacturer. Stained discs were then analysed with a Leica DM IRB confocal laser scanning microscope (Leica, Germany). Possible contaminations were also checked using the above described protocol.

\section{Statistical analysis}

Growth curves were obtained with the percentages of covered surface for each medium and each strain, obtained from the analysis of all the photographs. A linear mixedeffects model was used to make comparisons between the different observed growth curves. If $y_{i j}$ denotes the $j$-th observation of the $i$ strain then we assumes that: 


$$
y_{i j}=\beta_{1} t_{i j}+b_{1} t_{i j}+\varepsilon_{i j}
$$

where $t_{i j}$ is $j$-th observation time of the $i$-th strain. The parameter $\beta_{1}$ corresponds with the mean slope meanwhile the random variable $b_{1}$ (with normal distribution) corresponds with the random slope. It is assumed that $b_{1}$ has a normal distribution with null mean and standard deviation $\sigma_{b 1}$. The error term in the model, $\varepsilon_{i j}$ is assumed independent and normally distributed (with variance $\sigma_{2}$ ) for different times and different species. Additional details of the model used can be found in [9]. The statistical analysis was performed by using the NLME software package [10] and, particularly, for linear mixed-effect models, it has been used the package [11].

\section{Results}

\section{Sliding motility}

All strains with the exception of $M$. goodii, M. chelonae ATCC 19235, M. porcinum and M. septicum showed sliding motility on Middlebrook 7H9-0.3\% agar plates at the $7^{\text {th }}$ day (Figure 1). There were differences in the speed of sliding motility between strains, M. chelonae ATCC 35752T was found to be the slowest and M. abscessus the fastest (Figure 2D).

\section{Biofilm development}

The reproducibility of the test was confirmed using the $M$. smegmatis strains and testing them three times each. All three strains produced biofilm that covered almost identical percentages of the surface and had identical growth patterns. No difference in biofilm growth was found between this first reproducibility experiment and the following experiment where $M$. smegmatis strains were tested together with all NPRGM tested. Data reported here for $M$. smegmatis are those obtained in the experiment where all species were tested. All strains tested developed biofilm with all media.
The strains showed a sigmoid growth curve during the development of the biofilm (Figure 2A) with Middlebrook 7H9. M. abscessus was the fastest species, although no significant differences between them could be detected. The strains grew in Middlebrook 7 H9 showing initially a characteristic lace-like pattern, where bacteria were detected forming delicate nets of organisms (figure $3 \mathrm{~A})$. This pattern disappears when bacterial growth covers considerable parts of the surface (Figure $3 \mathrm{~B}$ ). All the strains covered $100 \%$ of the surface the $28^{\text {th }}$ day of incubation.

When sterile tap water was used as culture media, all the strains showed also a sigmoid growth curve (Figure 2B), however growth was slower and $100 \%$ of the surface was not covered until the day 63. Wells containing PBS-5\% glucose had the worst results for biofilm development because none of the tested strains grew enough to cover all the surface even by day 69 (Figure 2C).

Table 1 displays the models fitted. The first column (Middlebrook 7H9, PBS-5\% Glucose and Motility) indicates the data set meanwhile the model fitted is given in Equation 1. Basically, a random line passing through the origin plus a random error. The random slope has a normal distribution with mean $\beta_{\text {time }}$ and standard deviation $\sigma_{\text {time }}$.

The estimators for the different data sets appear in table 1 in the column headed $\beta_{\text {time }}$ and $\sigma_{\text {time }}$. Note that the standard deviation of this random slope for Middlebrook $7 \mathrm{H} 9$ is almost null and, in fact, we have a fixed slope estimates as 4.31 , i.e. for each additional day we observe a mean increment of $4.31 \%$ of covered surface for Middlebrook 7 H9.

Analogous interpretations can be given for the estimates of column $\beta_{\text {time }}$ corresponding to PBS-5\% Glucose and
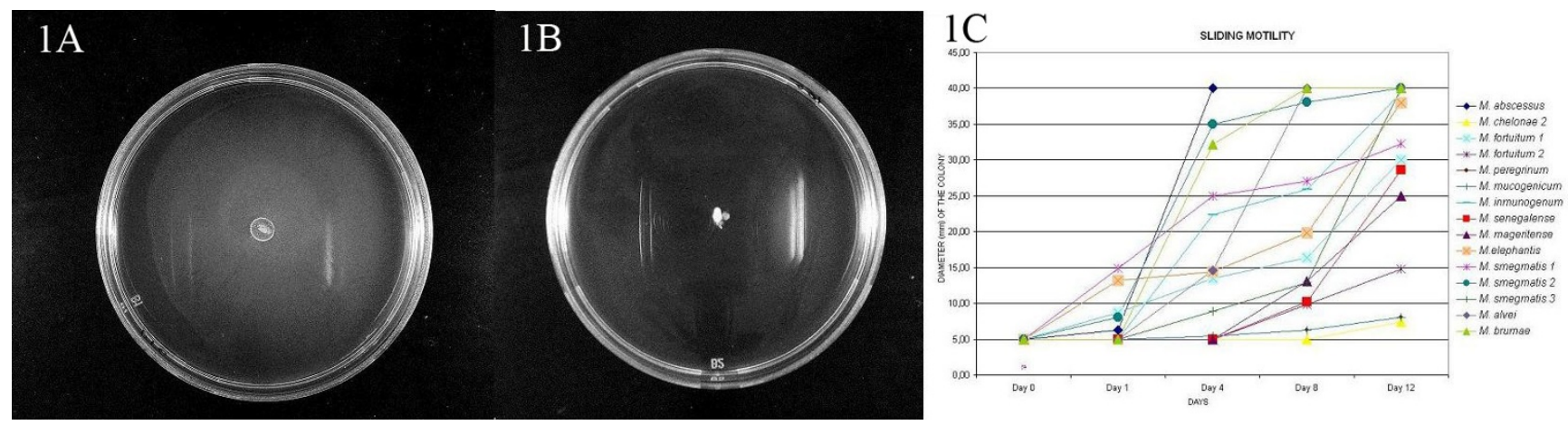

Figure I

Sliding motility of some strains. IA: M. abscessus (DSM 44I96T). IB: M. septicum (ATCC 70073 IT). IC: Diameter of the colony in the motility experiments. M. chelonae I: ATCC 19235;M. chelonae 2: ATCC 35752T; M. fortuitum I: ATCC 684 IT; M. fortuitum 2: ATCC 13756; M. smegmatis I: ATCC 607; M. smegmatis 2: ATCC 19420T; M. smegmatis 3: ATCC I4468. 


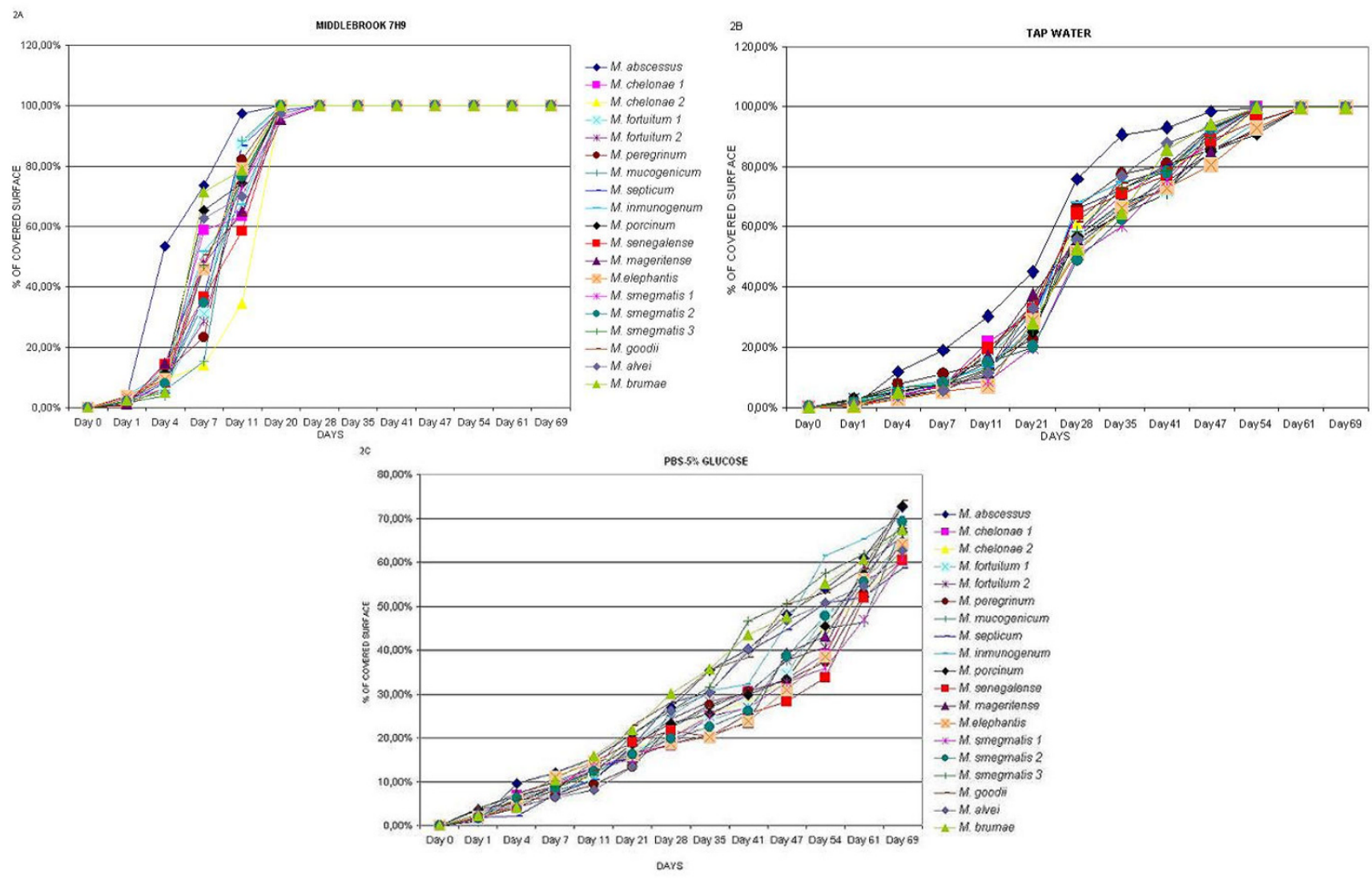

Figure 2

Growth on non-pigmented rapidly growing mycobacteria strains on the different media: $2 \mathrm{~A}$ : Middlebrook $7 \mathrm{H} 9$. 2B: Sterile tap water. 2C: PBS-Glucose 5\%. M. chelonae I: ATCC 19235; M. chelonae 2: ATCC 35752T; M. fortuitum I: ATCC 684IT; M. fortuitum 2: ATCC 13756; M. smegmatis I: ATCC 607; M. smegmatis 2: ATCC 19420T; M. smegmatis 3: ATCC 14468.

Motility (third and fourth rows). However, the mean increment is estimate as 0.88 for PBS-5\% Glucose and the corresponding standard deviation is estimated as 0.08 . For the motility the estimate is 2.25 but we have a large standard deviation estimated, 1.30. Additionally, the estimates of the standard deviation of the random error are shown in column headed $\sigma_{\text {residual }}$. Note the large standard deviation estimated for Middlebrook 7H9 and Motility.

It can be observed that the row corresponding to tap water has an additional column headed $\psi_{\text {type }}$. From the observation of the original data and by fitting a different linear model it was suggested that the behaviour of M. abscessus in this medium could be different from the other ones. We have introduced in the model an additional explanatory variable. The model now considered is:

$$
y_{i j}=\beta_{\text {time }} t_{i j}+\psi_{t y p e} t y p e+b_{1} t_{i j}+\varepsilon_{i j}
$$

where type $=1$ corresponds with $M$. abscessus and zero otherwise and $\psi_{\text {type }}$ is a constant. This model considers that the random slope can have a different mean for M. abscessus and a common but different mean for the other species. The mean of $M$. abscessus would be $\beta_{\text {time }}+\psi_{\text {type }}$ and for the other species would be $\beta_{\text {time }}$ respectively. This random slope will have a common standard deviation $\sigma_{\text {time }}$. We have compared, for the tap water the two models, given in equations 1 and 2. The p-value observed is lesser than 0.0001 , i.e. the mean of the random slope for M. abscessus is significatively different from the other species in tap water. In particular, the mean increment per unit time would be $1.78+9.25=11.02$ for M. abscessus and 1.77 for the other ones. Note that the standard deviation is almost null for the tap water data, i.e. we can consider a constant slope estimated with the values just commented. Finally a large standard deviation of the random error is estimated, 7.79 . 


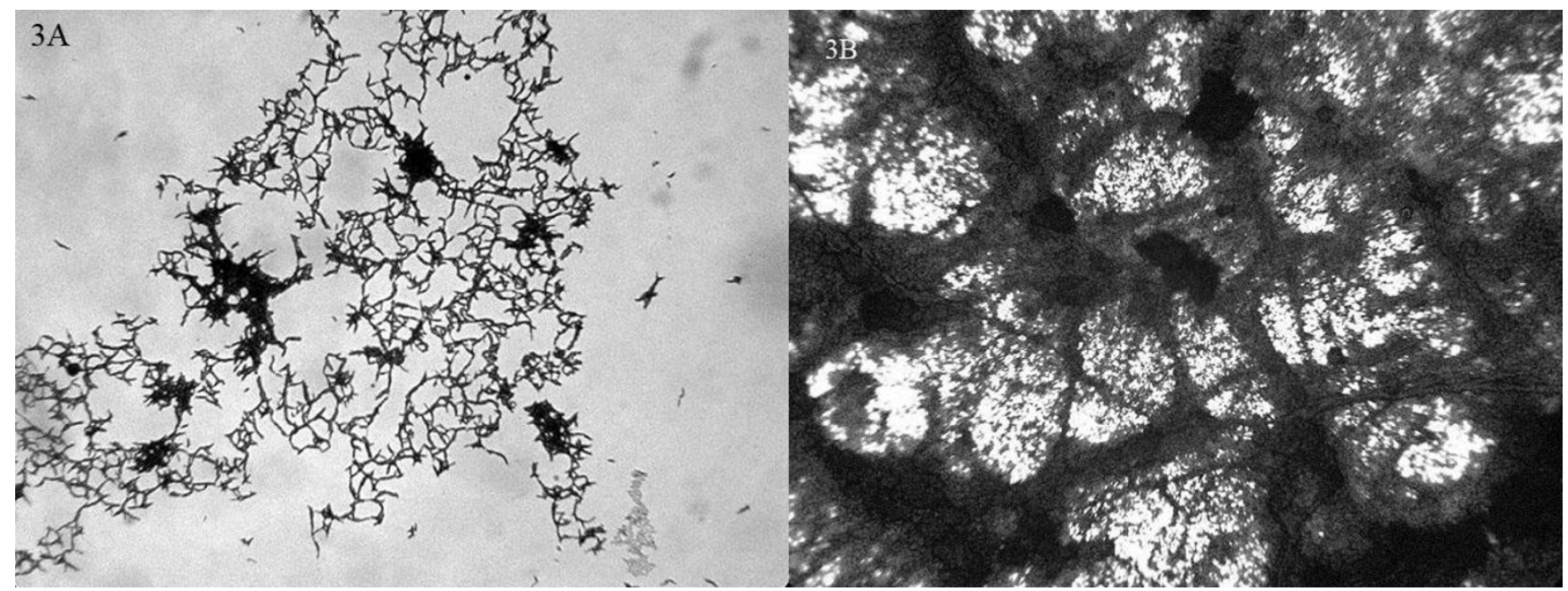

Figure 3

Morphology of biofilm development of $M$. fortuitum ATCC 684IT the day 4 (3A) and the day 24 (3B) in Middlebrook 7H9.

All the strains analysed by CLSM developed biofilm. The photographed structures showed identical growth patterns than those observed with fuchsine stain (Figure 4). Live/dead ${ }^{\circ}$ stain allow us to evaluate the presence of live and dead bacteria in the biofilm, with higher percentages of live bacteria in early states of biofilm development (75-80\% of live bacteria the day 7) than in the mature biofilm (approximately $50 \%$ of live bacteria the day 20 ). At day 24, biofilm had a thickness of around $20 \mu \mathrm{m}$ in all tested species.

\section{Discussion}

The relationship between biofilms and mycobacteria has been known for many decades, both in environmental and in medical settings. Detection of mycobacteria in biofilm samples from different water systems have been reported $[1,4,5,12-15]$. However, in these reports identification of the species was not achieved in all cases. Rapidly growing species, such as M. fortuitum and M. chelonae, have been described as part of these polymicrobial biofilms, where slowly growing mycobacteria have also been isolated. Recent taxonomic changes among this group of organisms, however, make the former identifications less valuable, because many of the recently characterized spe-

Table I: Summary of the fitted models.

\begin{tabular}{lllll}
\hline & $\sigma_{\text {time }}$ & $\sigma_{\text {residual }}$ & $\beta_{\text {time }}$ & $\beta_{\text {type }}$ \\
\hline Middlebrook 7H9 & $4.63 \mathrm{e}-05$ & 16.00 & 4.31 & \\
Tap water & $8.64 \mathrm{e}-06$ & 7.79 & 1.77 & 9.25 \\
PBS-5\% Glucose & 0.08 & 3.89 & 0.88 & \\
Motility & 1.30 & 5.87 & 2.24 & \\
\hline
\end{tabular}

cies were identified as M. chelonae or M. fortuitum according to old identification schemes [1].

The clinical significance of the isolates of NPRGM is unclear, with a high percentage of NPRGM being identified as "contaminants" or "colonizers", especially related with the origin of the sample [16]. Infections due to NPRGM include a broad range of diseases, the majority of them being caused by 3 of these species (M. fortuitum, $M$. chelonae and M. abscessus), with all other species rarely causing human infection [1,17-19], despite their relatively common isolation from clinical samples $[1,3,16,20,21]$. These pathogenic infections are commonly biofilmrelated ones, and include a large variety of surgical complications which ranged from relatively mild infections, such as surgical wound infections $[1,22]$, to extremely severe ones, such as prosthetic valve endocarditis $[23,24]$. In our hospital, biofilm-related infections represent half of the infections caused by NPRGM since 1980 to 2003 [3] and this percentage increased in a more recent multicenter study performed in our area [23]. Few reports evaluate in vitro biofilm development in NPRGM [8,25-36], although data concerning the detection of such mycobacteria in clinical samples has been published many years ago [37-40]. Recently, our laboratory described the ability of several strains of NPRGM to attach to polypropylene sutures, a first step in the development of biofilm [41]. Because biofilm development is a common pathogenic factor for many bacteria, and because the differences in clinical significance of the different species of NPRGM, we studied NPRGM of clinical interest to see if there were any differences in biofilm development. We found that all species tested can develop a biofilm, so this characteristic 


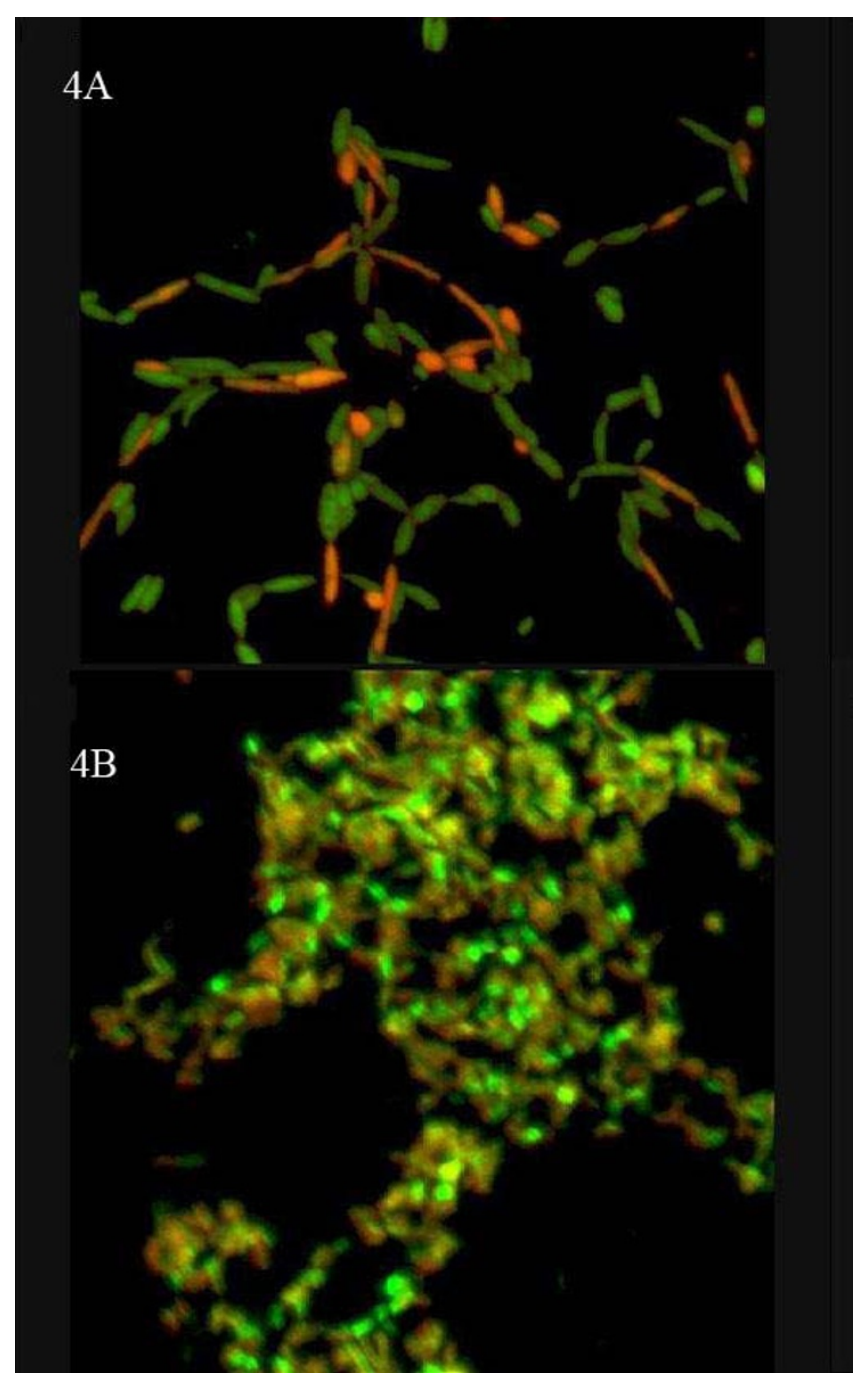

Figure 4

CLSM of mycobacterial biofilm. 4A: M. fortuitum (day 7, I00x), 4B: M. septicum (day 20 40x).

is not species-specific. However, we have found a faster growth of M. abscessus in one of the tested media. Because this species is probably the most pathogenic one $[1,2,16,42]$, this difference can represent a property that makes this species more pathogenic than others, despite all of tested NPRGM are able to develop a biofilm. $M$. abscessus has been described as the cause of many biofilmrelated diseases, and recent reports showed that the ability to develop biofilm seems to be related with other pathogenic mechanisms, such as colony phenotype or virulence in experimental models [29].

In this study biofilm development of representative NPRGM species was analyzed using several techniques. One of these techniques has been developed in our laboratory based in previously described systems [33]. It allows us to follow the dynamics of biofilm development, including an analysis of the morphology of the growing biofilm. For all NPRGM, a sigmoid growth curve was seen in two of the tested media, and a similar curve has been previously described for $M$. fortuitum using a different technique [27].

Another finding deals with the changes in biofilm development when using different media. A previous study [28] showed that low nutrient conditions decrease biofilm development for $M$. fortuitum and M. chelonae. In our study, we detected that such modifications in biofilm development could be detected among all the tested species. Tap water was found to be a better culture media than PBS-glucose for biofilm development. This difference can be due to the fact that tap water has many chemical molecules in minimal quantities that could be of great importance as nutrients for many organisms, including mycobacteria $[4,43]$. Another important issue could be the temperature. We select temperature for attachment based on a previous study performed by us [41], and we then select room temperature as the incubation temperature because it is the temperature where biofilms caused by these mycobacteria grows in natural environments. However, further experiments must be done to assess if temperature changes can affect the biofilm development. This factor can also influence the motility, because some species have also different optimal temperatures for growth. We selected $37^{\circ} \mathrm{C}$ for the motility experiment based on previously published studies [8].

Of interest, we have detected important differences in sliding motility among the studied strains, despite the fact that all of them can develop biofilm. Because these two properties have been linked previously $[8,29,31,32]$, and related with the presence of glycopeptidolipids in mycobacterial cell wall $[8,31]$, we speculate that there could be differences in the lipid content of the cell wall of the different species that can affect the results of the experiment. In our study, some representative strains did not show sliding motility, so it seems that this property is not always related with biofilm development, as has been recently described for Mycobacterium avium [44].

\section{Conclusion}

All the tested species of NPRGM are able to form biofilm in vitro, so this property is not species-specific in this group of mycobacteria. The chemical composition of the media influences the time necessary for the biofilm development of all tested species. The protocol described here allows is easy to perform and allows following the biofilm development and is useful for further studies in this field. 


\section{Authors' contributions}

JE coordinate all the study. Conceived and participate in the design the study, and participates in the analysis of the results. NZM perform the experimental study and collaborates in the analysis of the results. TJK participates in the analysis of the results and helped to draft the manuscript. GA perform the statistical analysis. RFR participates in the design of the study. IG participates in the design of the study and in the statistical analysis. All the authors participate in the redaction of the manuscript, have read it and approved it.

\section{Acknowledgements}

Nieves Z. Martín-de-Hijas was funded by the Fundación Conchita Rábago de Jiménez Díaz. Kinnari TJ was funded by the Academy of Finland, Paulo Foundation and Proteesisäätiö Foundation. This work was funded by a grant from the Comunidad de Madrid (S-0505/MAT/000324). Conflicts of interest: No conflicts for all authors.

\section{References}

I. Brown-Elliott BA, Wallace RJ: Clinical and Taxonomic Status of Pathogenic Nonpigmented or Late-Pigmenting Rapidly Growing Mycobacteria. Clinical Microbiology Reviews 2002, I 5(4):716-746.

2. De Groote MA, Huitt G: Infections due to rapidly growing mycobacteria. Clin Infect Dis 2006, 42: I756-I763.

3. Esteban J, Fernández-Roblas R, García-Cía Jl, Zamora N, Ortiz A: Clinical Significance and Epidemiology of Non-Pigmented Rapidly Growing Mycobacteria in a University Hospital. J Infect 2007, 54:135-145.

4. Vaerewijck MJM, Huys G, Palomino JC, Swings J, Portaels F: Mycobacteria in drinking water distribution systems: ecology and significance for human health. FEMS Microbiol Rev 2005, 29:9II-934.

5. Falkinham JO, Norton $C D$, LeChevallier MW: Factors influencing numbers of Mycobacterium avium, Mycobacterium intracellulare, and other mycobacteria in drinking water distribution systems. Appl Environ Microbiol 200I, 67(3): I225-I23I.

6. Donlan RM: Biofilms and device-associated infections. Emerging Infectious Diseases 200I, 7(2):277-279.

7. Fux CA, Costerton JW, Stewart PS, Stoodley P: Survival strategies of infectious biofilms. Trends Microbiol 2005, I 3(I):34-40.

8. Martinez A, Torello S, Kolter R: Sliding motility in mycobacteria. Journal of Bacteriology 1999, I8I(23):7331-7338.

9. Pinheiro JC, Bates DM: Mixed-Effects Models in S and S-PLUS. In Statistics and Computing Springer; 2000.

10. R-Development-Core-Team: R: A Language and Environment for Statistical Computing Vienna: R Foundation for Statistical Computing; 2006.

11. Pinheiro JC, Bates DM, DebRoy S, Sarkar D: NLME: Linear and nonlinear mixed effects models. $R$ package version 3.I-79. edn 2006.

12. Torvinen E, Suomalainen S, Lehtola MJ, Miettinen IT, Zacheus O, Paulin L, Katila ML, Martikainen PJ: Mycobacteria in water and loose deposits of drinking water distribution systems in Finland. Appl Environ Microbiol 2004, 70(4): 1973-198I.

13. Wallace RJ, Musser JM, Hull SI, Silcox VA, Steele LC, Forrester GD, Labidi A, Selander RK: Diversity and sources of rapidly growing mycobacteria associated with infections following cardiac surgery. J Infect Dis 1989, I59(4):708-716.

14. Santos R, Oliveira F, Fernandes J, Gonçalves S, Macieira F, Cadete M: Detection and identification of mycobacteria in the Lisbon water distribution system. Water Sci Technol 2005, 52(8): $177-180$.

15. Barbeau J, Gauthier C, Payment P: Biofilms, infectious agents, and dental unit waterlines: a review. Can J Microbiol 1998, 44(II): $1019-1028$.

16. Esteban J, Martín-de-Hijas NZ, Fernández Al, Fernández-Roblas R, Gadea I, Madrid-Study-Group-of-Mycobacteria: Epidemiology of infections due to Non-pigmented Rapidly Growing Mycobac- teria diagnosed in an urban area. Eur J Clin Microbiol Infect Dis 2008, 27(10):95I-7.

17. Sohail MR, Smilack JD: Hernia repair mesh-associated Mycobacterium goodi infection. J Clin Microbiol 2004, 42(6):2858-2860.

18. Schinsky MF, McNeil MM, Whitney AM, Steigerwalt AG, Lasker BA, Floyd MM, Hogg GG, Brenner DJ, Brown JM: Mycobacterium septicum sp. nov., a new rapidly growing species associated with catheter-related bacteraemia. Int J Syst Evol Microbiol 2000, 50(Pt 2):575-58I.

19. Schinsky MF, Morey RE, Steigerwalt AG, Douglas MP, Wilson RW, Floyd MM, Butler WR, Daneshvar MI, Brown-Elliott BA, Wallace RJ: Taxonomic variation in the Mycobacterium fortuitum third biovariant complex: Description of Mycobacterium boenickei sp. nov., Mycobacterium houstonense sp. nov., Mycobacterium neworleansense sp. nov., and Mycobacterium brisbanense sp. nov., and recognition of Mycobacterium porcinum from human clinical specimens. Int J Syst Evol Microbiol 2004, 54(Pt 5):1653-1667.

20. Burns DN, Wallace RJ, Schultz ME, Zhang YS, Zubairi SQ, Pang YJ, Gibert CL, Brown BA, Noel ES, Gordin FM: Nosocomial outbreak of respiratory tract colonization with Mycobacterium fortuitum : demonstration of the usefulness of pulsed-field gel electrophoresis in an epidemiologic investigation. Am Rev Respir Dis 1991, 144(5): I I53-1159.

21. Wallace RJ, Brown BA, Griffith DE: Nosocomial outbreaks/ pseudo-outbreaks caused by nontuberculous mycobacteria. Annu Rev Microbiol 1998, 52:453-490.

22. Wallace RJ, Swenson JM, Silcox VA, Good RC, Tschen JA, Stone MS: Spectrum of disease due to rapidly growing mycobacteria. Rev Infect Dis 1983, 5(4):657-679.

23. Kuritsky JN, Bullen MG, Broome CV, Silcox VA, Good RC, Wallace $\mathrm{RJ}$ : Sternal wound infections and endocarditis due to organisms of the Mycobacterium fortuitum complex. Ann Intern Med 1983, 98(6):938-939.

24. Olalla J, Pombo M, Aguado JM, Rodriguez E, Palenque E, Costa JR, Rioperez E: Mycobacterium fortuitum complex endocarditiscase report and literature review. Clin Microbiol Infect 2002, 8(2): $125-129$.

25. Bardouniotis $\mathrm{E}, \mathrm{Ceri} \mathrm{H}$, Olson ME: Biofilm formation and biocide susceptibility testing of Mycobacterium fortuitum and Mycobacterium marinum. Curr Microbiol 2003, 46:28-32.

26. Chen JM, German G], Alexander DC, Ren H, Tan T, Liu J: Roles of Lsr2 in colony morphology and biofilm formation of Mycobacterium smegmatis. Journal of Bacteriology 2006, I 88(2):633-64I.

27. Hall-Stoodley L, Lappin-Scott H: Biofilm formation by the rapidly growing mycobacterial species Mycobacterium fortuitum. FEMS Microbiol Lett 1998, 168(1):77-84.

28. Hall-Stoodley L, Keevil CW, Lappin-Scott H: Mycobacterium fortuitum and Mycobacterium chelonae biofilm formation under high and low nutrient conditions. J Appl Microbiol I999, 65(Symposium Supplement):60S-69S.

29. Howard ST, Rhoades E, Recht J, Pang X, Alsup A, Kolter R, Lyons CR, Byrd TF: Spontaneous reversion of Mycobacterium abscessus from a smooth to a rough morphotype is associated with reduced expression of glycopeptidolipid and reacquisition of an invasive phenotype. Microbiology 2006, 152:158I-1590.

30. Ojha A, Anand M, Bhatt A, Kremer L, Jacobs WR, Hatfull GF: GroELI: A dedicated chaperone involved in mycolic acid biosynthesis during biofilm formation in mycobacteria. Cell 2005, I 23:86 I-873.

31. Recht J, Kolter R: Glycopeptidolipid acetylation affects sliding motility and biofilm formation in Mycobacterium smegmatis. J Bacteriol 200I, 183(19):5718-5724.

32. Recht J, Martinez A, Torello S, Kolter R: Genetic analysis of sliding motility in Mycobacterium smegmatis. Journal of Bacteriology 2000, I 82(15):4348-435I.

33. Rose L, Kaufmann SH, Daugelat S: Involvement of Mycobacterium smegmatis undecaprenyl phosphokinase in biofilm and smegma formation. Microbes Infect 2004, 6(I I):965-97I.

34. Teng R, Dick T: Isoniazid resistance of exponentially growing Mycobacterium smegmatis biofilm culture. FEMS Microbiol Lett 2003, 227: $17 \mid-174$.

35. Zambrano MM, Kolter R: Mycobacterial biofilms: a greasy way to hold it together. Cell 2005, I 23:762-764.

36. Greendyke R, Byrd TF: Differential antibiotic susceptibility of Mycobacterium abscessus variants In biofilms and macro- 
phages compared to planktonic bacteria. Antimicrob Agents Chemother 2008, 52(6):2019-2026.

37. Schulze-Robbecke R, Feldmann C, Fischeder R, Janning B, Exner M, Wahl G: Dental units: an environmental study of sources of potentially pathogenic mycobacteria. Tuber Lung Dis 1995, 76(4):318-323.

38. Schulze-Robbecke R, Janning B, Fischeder R: Occurrence of mycobacteria in biofilm samples. Tuber Lung Dis 1992, 73(3): |4|-| 44.

39. Schulze-Röbbecke R, Fischeder R: Mycobacteria in biofilms. Zentralbl Hyg Umweltmed 1989, 188(3-4):385-390.

40. Raad II, Vartivarian S, Khan A, Bodey GP: Catheter-related infections caused by the Mycobacterium fortuitum complex: 15 cases and review. Rev Infect Dis | 99 |, I 3(6): I I20-I I 25.

41. Zamora N, Esteban J, Kinnari TJ, Celdran A, Granizo JJ, Zafra C: In vitro evaluation of the attachment to polypropylene sutures on non-pigmented rapidly growing mycobacteria. Clin Microbiol Infect 2007, 13(9):902-907.

42. Petrini B: Mycobacterium abscessus : An emerging rapid-growing potential pathogen. APMIS 2006, I | 4:3| 9-328.

43. Chalupka S: Tainted water on tap: What to tell patients about preventing illness from drinking water. Am J Nurs 2005, 105(II):40-52.

44. Freeman R, Geier H, Weigel KM, Do J, Ford TE, Cangelosi GA: Roles for Cell Wall Glycopeptidolipid in Surface Adherence and Planktonic Dispersal of Mycobacterium avium. Appl Environ Microbiol 2006, 72(I 2):7554-7558.

Publish with Biomed Central and every scientist can read your work free of charge

"BioMed Central will be the most significant development for disseminating the results of biomedical research in our lifetime. "

Sir Paul Nurse, Cancer Research UK

Your research papers will be:

- available free of charge to the entire biomedical community

- peer reviewed and published immediately upon acceptance

- cited in PubMed and archived on PubMed Central

- yours - you keep the copyright 